\title{
Differentiation of Crohn's disease and ulcerative colitis using intestinal wall thickness of the colon: A Diagnostic accuracy study of endoscopic ultrasonography
}

\author{
Nader Roushan ${ }^{1}$, Nasser Ebrahimi Daryani ${ }^{2}$, Zahra Azizi $^{3}$, Helia Pournaghshband ${ }^{4}$, Ali Niksirat*5 \\ Received: 26 Sep 2018 \\ Published: 19 Jun 2019
}

\begin{abstract}
Background: The differentiation between Ulcerative Colitis (UC) and Crohn's Disease (CD) is an important issue for choosing the appropriate treatment. Endoscopic Ultrasonography (EUS) has been used to distinguish different layers of the gastrointestinal wall. We performed this study to evaluate the accuracy of EUS in differentiating colonic UC from CD compared to standard tests (colonoscopy,
\end{abstract} pathology, imaging, and clinical presentation).

Methods: This is a prospective, single-blinded diagnostic accuracy study, on 70 patients (30 UC, $30 \mathrm{CD}$, and 10 healthy controls) After obtaining informed consent, patients underwent a complete workup and were referred to an endosonographist who was blind to the diagnosis. The thickness of mucosa, submucosa and the total wall (TWT) of mid-sigmoid colon were measured by Pentax radial echoendoscope EPKI-7000 with Avius Hitachi ultrasound system (Japan). Statistical analyses were performed using the SPSS statistical software (v23). Statistical significance was considered if P-values were less than 0.05 .

Results: Our study revealed a sensitivity of $100 \%$ (90.7-100\%) and specificity of $90.9 \%$ (70.8-98.8\%) for EUS to differentiate UC and CD compared to standard diagnostic tests. Mean mucosal thickness in patients with UC was significantly greater than patients with $\mathrm{CD}$, while, the mean sub-mucosal thickness was significantly greater in patients with $\mathrm{CD}(\mathrm{p}<0.001)$. The sensitivity and specificity of mean mucosal thickness for differentiating UC from $\mathrm{CD}$ and controls were $92.3 \%$ and $88.6 \%$ with a cut-off point of $1.1 \mathrm{~mm}(\mathrm{p}<0.001$ ). Moreover, sensitivity and specificity of mean submucosal thickness for differentiating CD from UC and controls were $100 \%$ and $86.1 \%$ with a cut-off point of $1.08 \mathrm{~mm}(\mathrm{p}<0.001)$.

Conclusion: EUS can be used as an efficient modality with acceptable accuracy to differentiate Crohn's disease and Ulcerative Colitis and to determine disease activity.

Keywords: Endoscopic ultrasonography, Intestinal wall thickness, Sensitivity, Specificity, Crohn's disease, Ulcerative colitis

Conflicts of Interest: None declared

Funding: Institutional Ethics Committee of Tehran University of Medical Sciences

*This work has been published under CC BY-NC-SA 1.0 license.

Copyright $₫$ Iran University of Medical Sciences

Cite this article as: Roushan N, Ebrahimi Daryani N, Azizi Z, Pournaghshband H, Niksirat A. Differentiation of Crohn's disease and ulcerative colitis using intestinal wall thickness of the colon: A Diagnostic accuracy study of endoscopic ultrasonography. Med J Islam Repub Iran. 2019 (19 Jun);33:57. https://doi.org/10.47176/mjiri.33.57

Introduction

Inflammatory Bowel Disease (IBD) is a chronic and re- lapsing intestinal disorder mediated by the immune sys-

Corresponding author: Dr Ali Niksirat, a-niksirat@razi.tums.ac.ir

1. Department of internal medicine, Division of Gastroenterology, Imam Khomeini Hospital, Tehran University of Medical Sciences, Tehran, Iran

2. Department of internal medicine, Division of Gastroenterology, Tehran

University of Medical Sciences, Tehran, Iran

3. Faculty of Health, York University, Toronto, Canada

4. Medical Student, Department of Medicine, Iran University of Medical

Sciences, Tehran, Iran

5. Internist, Tehran University of Medical Sciences, Tehran, Iran $\uparrow$ What is "already known” in this topic:

While there is no single standard method for diagnosing Ulcerative Colitis (UC) and Crohn's Disease (CD), ultrasonography (US) has been described as a non-invasive, less costly and efficient method that can be used for diagnosis and follow up of patients with IBD.

\section{$\rightarrow$ What this article adds:}

The sensitivity and specificity of EUS were $100 \%$ and $90.9 \%$ in our study. The sensitivity and specificity of mean mucosal thickness for differentiating UC form CD and Controls were $92.3 \%$ and $88.6 \%$ with a cut-off point of $1.1 \mathrm{~mm}$; while, sensitivity and specificity of mean submucosal thickness for differentiating CD from UC and Controls were $100 \%$ and $86.1 \%$ with a cut-off point of $1.08 \mathrm{~mm}$. The thickness of colonic layers was also associated with disease activity indices, which shows the accuracy of this diagnostic method in determining disease activity for patients' follow up, in addition to its diagnostic usage. 
tem. Two main subtypes of IBD are Ulcerative Colitis (UC), an inflammatory mucosal disease, frequently involving the rectum and extending proximally, that can involve the entire colon, and Crohn's Disease (CD), a transmural inflammation of gastrointestinal (GI) mucosa, involving any part of it from oral cavity to anus $(1,2)$. Differentiating between these two conditions is an important issue for choosing the appropriate medical treatment, determination the time of surgery and estimating prognosis (3).

Involvement of any part of the GI tract other than colon and/or fistula formation defines Crohn's disease. Inflammation of colon per se can be seen in both UC and CD. Some colonoscopic findings such as linear ulcers, cobblestone and skip lesions are suggestive of CD, but these findings are not pathognomonic and not seen commonly. Biopsy during colonoscopy only defines Crohn's disease in a small number of cases, and most of the times, it is non-diagnostic. However, multiple biopsies from different locations are recommended to increase the diagnostic accuracy. There is no single standard method for diagnosing $\mathrm{CD}$, and change in diagnosis of UC during the first year occurs in about $5 \%$ of cases $(4,5)$.

Endoscopic Ultrasonography (EUS) is a modality to differentiate different layers of the gastrointestinal wall $(6$, 7). Recently, this tool has been used to assess the activity of IBD, fistulae, abscesses, and regional lymphadenopathies (8-11). Nonetheless, its accuracy compared to colonoscopy in differentiating UC and CD needs to be studied. Therefore, we performed this study to evaluate the sensitivity, specificity of EUS compared to standard diagnostic tests (Colonoscopy, pathology, imaging, and clinical presentation), and its accuracy to differentiate $\mathrm{CD}$ and UC.

\section{Methods \\ Study population}

This is a prospective, single-blinded diagnostic accuracy study approved by the Institutional Ethics Committee of Tehran University of Medical Sciences (TUMS). The study was performed between 2016-2017 in Imam Khomeini Hospital, Tehran, Iran. Patients were selected using a simple sampling method. UC group consisted of 30 patients, $18(60 \%)$ male, with mean age of $36.2 \pm 10.97$ (2464) years and the CD group included 30 patients with Crohn's disease (CD), 13(43.3\%) male patients with a mean age of $34.36 \pm 8.13(20-52)$. Ten healthy people including $6(60 \%)$ male patients, with a mean age of $41.4 \pm 11.51$ (29-62) years were selected as the control group. After obtaining informed consent, patients underwent a complete workup including history and physical examination, clinical assessment of disease activity indexes by means of Crohn's Disease Activity Index (CDAI) and UC Mayo Score, measuring inflammatory markers including erythrocyte sedimentation rate (ESR), Creactive protein (CRP), and calprotectin. Patients who were diagnosed to have IBD by a gastroenterologist using standard diagnostic methods (colonoscopy, pathology, imaging, clinical presentation, and inflammatory markers) and healthy controls without IBD were referred to an endosonographist who was blind to the diagnosis.

\section{Primary Endpoints}

The primary endpoint of this study was to assess sensitivity and specificity of EUS to differentiate UC from CD in contrast to standard diagnostic tests (Colonoscopy, Pathology, imaging and clinical presentation) by means of sigmoid colon wall layers thickness.

\section{Diagnosis criteria \& Disease Severity Assessment}

To assess disease activity and severity of UC, we used Mayo disease activity score, which is a combined endoscopic and clinical score (consisting of 4 sub-categories: frequency of defecation, bleeding during defecation, findings of colonoscopy and physician overall assessment that ranges from 0 to 12) (12).

The Crohn's Disease Activity Index (CDAI) was used to assess disease severity in patients with $\mathrm{CD}$ consisting of 8 factors of patients' symptoms during previous seven days and clinical data that are adjusted with a weighting factor and then summed. The score ranges from 0 to 600 (13).

The disease was considered clinically active if the CDAI score was higher than 150 and Mayo Score was higher than $3(12,13)$. All patients with UC had active disease, while only $86.7 \%$ of patients with CD had Active disease.

\section{Inclusion and Exclusion criteria}

The Inclusion criteria consisted of confirmed CD and UC by standard diagnostic tests and age $>18$ years. The exclusion criteria of the study were acute bowel obstruction or significant stenosis, acute infection, endoluminal tumors, acute diverticulitis, and fistula. Patients in the control group were selected if they had no sign of macroscopic and clinical colitis and if they didn't have any family history of IBD. Indication of EUS in the control group was the presence of any disorders other than IBD, such as submucosal lesions.

\section{Procedure Method}

Patients were recommended to have clear liquid dietary regimen 48 hours before the procedure and were prescribed 5mg Bisacodyl tablets (Tolid Darou, Tehran, IRAN) every 6 hours and 6-8 boxes of Polyethylene glycol (PEG) (each bag 70-gram solved in 1 liter water) until clearance of rectal output on the day before the procedure.

EUS was performed with a Pentax radial echoendoscope EPKI-7000 with Avius Hitachi ultrasound system (Japan). The thickness of mucosal, submucosal layers and total wall of the mid-sigmoid colon were measured in all cases by one endosonographist. In order to assess the reliability of EUS to assess the thickness of sigmoid wall layers, measurements were repeated in random areas for 3 different areas and mean values were used for analysis.

\section{Statistical Analysis}

Reliability analysis of EUS: For determining the reliability of EUS results, test-retest method was used for analyzing the data. All measurements were repeated 3 times in randomly selected areas of the mid-sigmoid re- 
gion. If the Pearson correlation coefficient (r) was more than 0.7 and the p-value was under 0.05 , the test was considered reliable.

Overall Analysis: Descriptive statistics were reported using frequency (percentage) for categorical variable and mean \pm standard deviation for continuous variable. Sensitivity, specificity, positive predictive value (PPV), negative predictive value (NPV) of EUS in differentiating CD from UC were calculated to assess the accuracy of EUS versus standard method. The thickness of different layers of intestine was compared between $\mathrm{UC}, \mathrm{CD}$, and controls with independent sample t-test. Sensitivity and specificity and cut-off point of the thickness of different intestinal layers to differentiate $\mathrm{CD}$ and $\mathrm{UC}$ from controls and $\mathrm{CD}$ from UC were calculated using Roc curve analysis. The optimal cut-off point was reached via consensus between 2 analyzers and via Medcalc software. Correlation analysis was used to assess the correlation between inflammatory markers and severity index and thickness of different layers. P-value of less than 0.05 was considered statistically significant. All the statistical analyses were performed using the SPSS statistical software (v23).

\section{Results}

Overall, 26 patients were diagnosed with Ulcerative Colitis (UC), and 34 were diagnosed with Crohn's Disease (CD). Descriptive results of patients' demographics and clinical data are summarized in Table 1.

After performing EUS on patients through blinded examination, 6 patients in the UC group were diagnosed as CD. However, the result of EUS in all patients of the CD group was consistent with the standard tests. We performed MR-Enterography on patients with a new diagno- sis, which confirmed CD diagnosis in 4 patients (True Positive: 4+34, False Positive: 2, True Negative: 20, False Negative: 0 ).

Endoscopic Ultrasonography (EUS) showed sensitivity and specificity of $100 \%(90.7-100 \%)$ and $90.9 \%(70.8-$ $98.8 \%$ ) for differentiating CD from UC compared to the gold standard technique, respectively (Table 2).

\section{Reliability and accuracy of EUS in diagnosing UC and $C D$}

According to the result of EUS and using the test-retest method, our data showed reliable results during 3 measurements of mucosal, submucosal and total wall thickness of mid-sigmoid walls.

Average Pearson correlation coefficient (r) was calculated for each layer. Mean Pearson correlation coefficient (r) was $0.73,0.80$ and 0.75 for mucosal, submucosal and total wall thickness, respectively; which showed reliable results.

\section{Thickness of intestine layers}

Mean mucosal, and total wall thickness (TWT) in UC patients were significantly greater than the controls (MT: Mean Difference $(\mathrm{MD})=1.2 \pm 0.1$, TWT: $\mathrm{MD}=1.37 \pm 0.2$, $\mathrm{p}<0.001)$. However, mean sub-mucosal thickness didn't show any significant difference $(\mathrm{ST}: \mathrm{MD}=0.24 \pm 0.1$, $\mathrm{p}=0.160$ ).

Mean sub-mucosal and TWT in patients with CD were significantly greater than controls $(\mathrm{ST}: \mathrm{MD}=1.18 \pm 0.2$, TWT: $\mathrm{MD}=1.61 \pm 0.2, \mathrm{p}<0.001)$. However, mean mucosal thickness didn't show any significant difference (MT: $\mathrm{MD}=0.25 \pm 0.1, \mathrm{p}=0.090)$.

Mean mucosal thickness in patients with UC was signif-

Table 1. Demographic and clinical characteristic of patients

\begin{tabular}{|c|c|c|c|}
\hline & $\mathrm{UC}(\mathrm{n}=26)$ & $\mathrm{CD}(\mathrm{n}=34)$ & Controls $(n=10)$ \\
\hline Age, Mean \pm SD & $35.69 \pm 10.68$ & $34.97 \pm 8.87$ & $41.4 \pm 11.51$ \\
\hline Sex (Male), n (\%) & $15(57.7 \%)$ & $16(47.1 \%)$ & $6(60 \%)$ \\
\hline CDAI, Mean \pm SD & - & $87.18 \pm 46.62$ & - \\
\hline Mayo Score, Mean \pm SD & $7.61 \pm 1.91$ & - & - \\
\hline \multirow[t]{3}{*}{ Extension of disease, $\mathrm{n}(\%)$} & Left Colitis: 3 (11.5\%) & Colitis: $15(44.1 \%)$ & - \\
\hline & Pan-Colitis: $21(80.8 \%)$ & Ileocolitis: $15(44.1 \%)$ & \\
\hline & Extensive: $2(7.7 \%)$ & & \\
\hline $\mathrm{ESR}$, Mean $\pm \mathrm{SD}$ & $20.69 \pm 12.05$ & $19.58 \pm 14.40$ & - \\
\hline $\mathrm{CRP}$, Mean $\pm \mathrm{SD}$ & $13.62 \pm 34.04$ & $11.26 \pm 17.03$ & - \\
\hline Calprotectin, Mean \pm SD & $115.96 \pm 116.61$ & $128.38 \pm 106.41$ & - \\
\hline Mean Mucosal Thickness, Mean \pm SD & $1.8 \pm 0.59$ & $0.83 \pm 0.45$ & $0.58 \pm 0.17$ \\
\hline Mean Sub-Mucosal Thickness, Mean \pm SD & $0.92 \pm 0.52$ & $1.86 \pm 0.68$ & $0.68 \pm 0.12$ \\
\hline Mean Total Wall Thickness, Mean \pm SD & $3.6 \pm 0.69$ & $3.89 \pm 0.77$ & $2.28 \pm 0.39$ \\
\hline \multicolumn{4}{|c|}{$\begin{array}{l}\text { *Abbreviations: SD: Standard Deviation, CDAI: Clinical Disease Activity Index, ESR: Erythrocyte Sedimentation Rate, CRP: C-reactive protein, UC: Ulcerative Colit } \\
\text { CD: Crohn's Disease }\end{array}$} \\
\hline & Sensitivity & Specificity & Cutoff \\
\hline $\begin{array}{l}\text { Overall EUS accuracy in differentiating CD from } \\
\text { UC compared to gold standard }\end{array}$ & $100 \%(90.7-100 \%)$ & $90.9 \%(70.8-98.8 \%)$ & - \\
\hline $\begin{array}{l}\text { Mean Mucosal Thickness for differentiating UC } \\
\text { form CD and Controls }\end{array}$ & $92.3 \%$ & $88.6 \%$ & $1.1 \mathrm{~mm}$ \\
\hline $\begin{array}{l}\text { Mean Sub-Mucosal Thickness for differentiating CD } \\
\text { from UC and Controls were }\end{array}$ & $100 \%$ & $86.1 \%$ & $1.08 \mathrm{~mm}$ \\
\hline TWT for differentiating IBD from controls & $98.3 \%$ & $100 \%$ & $2.75 \mathrm{~mm}$ \\
\hline TWT for differentiating CD from Controls & $97.1 \%$ & $100 \%$ & $2.91 \mathrm{~mm}$ \\
\hline TWT for differentiating CD from UC and Control & $70.6 \%$ & $58.3 \%$ & $3.46 \mathrm{~mm}$ \\
\hline
\end{tabular}


icantly greater than patients with $\mathrm{CD}(\mathrm{MD}=1.03 \pm 0.13$, $\mathrm{p}<0.001$ ), while, mean sub-mucosal thickness was significantly greater in patients with $\mathrm{CD}$ than UC patients $(\mathrm{MD}=$ $0.94 \pm 0.16, p<0.001)$. However, mean TWT wasn't significantly different between $\mathrm{UC}$ and $\mathrm{CD}$ patients $(\mathrm{MD}=$ $0.2 \pm 0.19, \mathrm{P}=0.200$ ) (Figs. $1 \& 2$ ).

Sensitivity and specificity of intestinal layers thickness for differentiating UC and CD

Sensitivity and specificity of mean mucosal thickness for differentiating UC form CD and controls were $92.3 \%$ and $88.6 \%$ with a cut-off point of $1.1 \mathrm{~mm}$ (Area Under Curve $=0.94(95 \% \mathrm{CI}=0.88-1), \mathrm{p}<0.001)$. (Table 2, Fig. 3).

Moreover, sensitivity and specificity of mean submucosal thickness for differentiating $\mathrm{CD}$ from UC and controls were $100 \%$ and $86.1 \%$ with a cut-off point of $1.08 \mathrm{~mm}$ (Area Under Curve $=0.94(95 \% \mathrm{CI}=0.88-1)$, $\mathrm{p}<0.001$ ) (Table 2, Fig. 4).
Sensitivity and specificity of TWT for differentiating IBD from controls were $98.3 \%$ and $100 \%$ with a cut-off point of $2.75 \mathrm{~mm}$. (Area Under Curve $=0.98(95 \% \mathrm{CI}=$ $0.96-1), p<0.001)$. While, the sensitivity and specificity of TWT for differentiating CD from controls were 97.1 and $100 \%$ with a cut-off point of $2.91(\mathrm{AUC}=0.97(95 \% \mathrm{CI}=$ $0.93-1), \mathrm{p}<0.001)$ and for differentiating $\mathrm{CD}$ from UC and control were 70.6 and $58.3 \%$ with a cut-off point of 3.46 $\mathrm{mm}(\mathrm{AUC}=0.72(95 \% \mathrm{CI}=0.6-0.84), \mathrm{p}=0.001)($ Table 2$)$.

Correlation between intestinal layers thickness and Disease Activity Index and inflammatory markers

TWT was significantly correlated with Mayo score in patients with $\mathrm{UC}(\mathrm{r}=0.59, \mathrm{p}=0.001)$.

Furthermore, submucosal thickness was significantly correlated with CDAI $(\mathrm{r}=0.66, \mathrm{p}=0.001)$, ESR $(\mathrm{r}=0.51$, $\mathrm{p}=0.002)$, CRP $(\mathrm{r}=0.42, \mathrm{p}=0.010)$, and Calprotectin $(\mathrm{r}=0.66, \mathrm{p}=0.001)$ in patients with $\mathrm{CD}$.

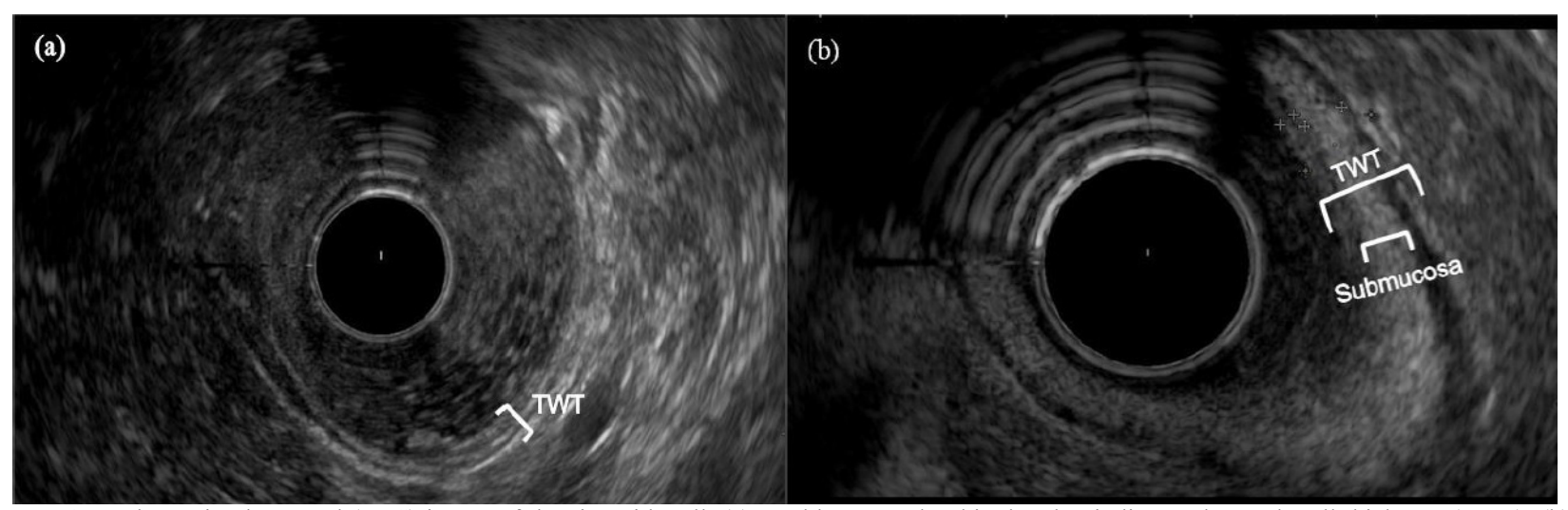

Fig. 1. Endoscopic ultrasound (EUS) image of the sigmoid wall. (a) Healthy control; white bracket indicates the total wall thickness (TWT). (b) Patient with active Crohn's disease; the larger white bracket indicates TWT and the smaller white bracket comprises the enlarged submucosal layer

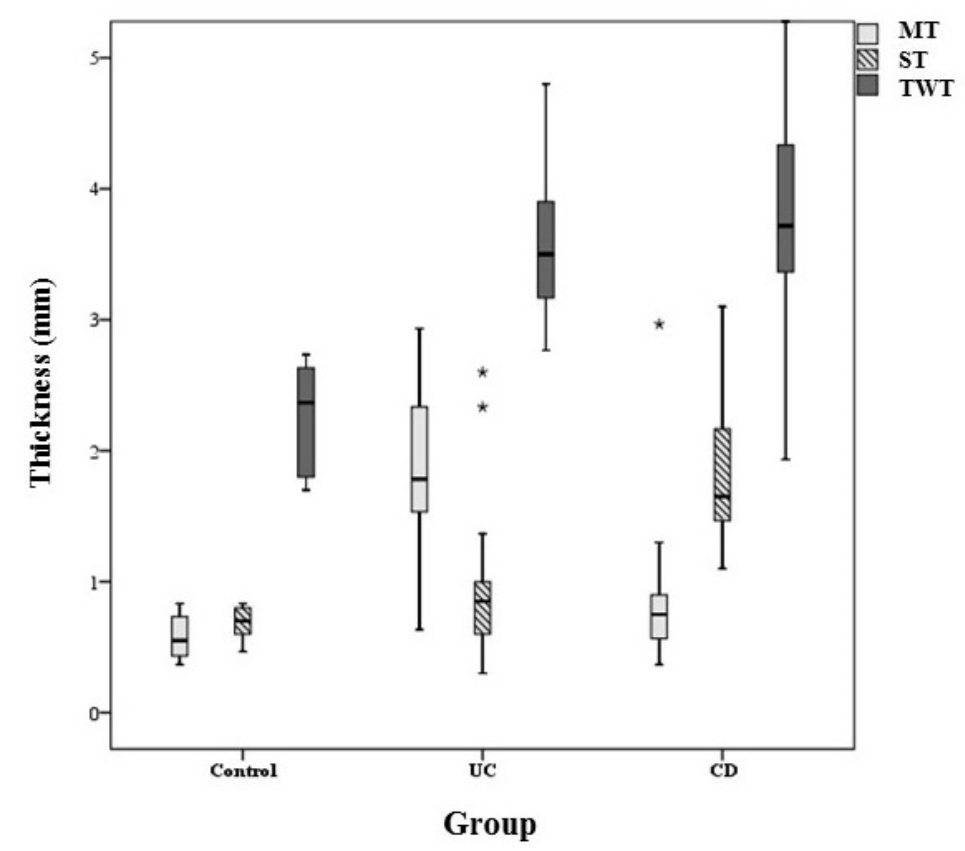

Fig. 2. *Box Plot of mucosal, sub-mucosal and total wall thickness in patients with Ulcerative Colitis, Crohn's Disease, and Controls. Each Box represents minimum, first quartile, median, third quartile, and maximum of thickness in each layer. ** MT: Mucosal Thickness, ST: Sub-Mucosal Thickness, TWT: Total Wall Thickness. 


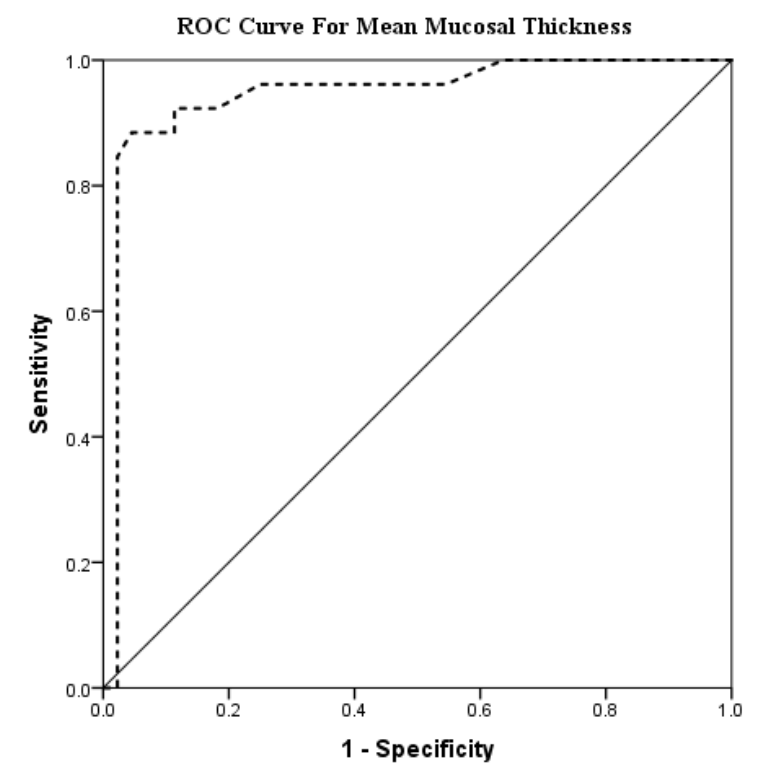

Fig. 3. ROC Curve, demonstrating sensitivity and 1-Specificity of Mean Mucosal Thickness for differentiating Ulcerative Colitis form Crohn's Disease and Controls

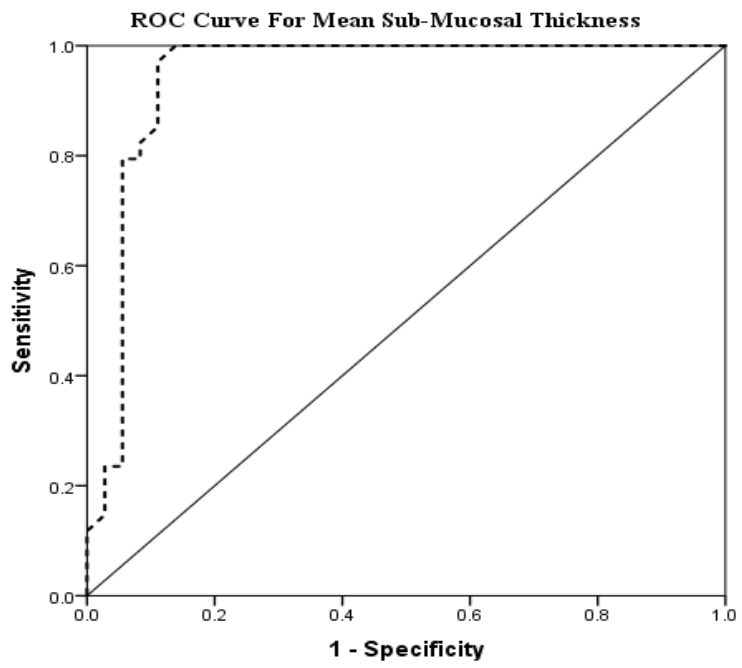

Fig. 4. ROC Curve, demonstrating Sensitivity and 1-Specificity of Mean Sub-Mucosal Thickness for differentiating Crohn's Disease from Ulcerative Colitis and Controls.

\section{Discussion}

This study presented acceptable accuracy of EUS with reliable results versus standard tests (colonoscopy, pathology, imaging, and clinical presentation) in the diagnosis of CD and UC (sensitivity: 100\%, specificity: 90.9\%). Moreover, the thickness of different layers of intestine could differentiate UC and $\mathrm{CD}$ and was significantly correlated with disease activity index and inflammatory markers.

Ultrasonography (US) has been described as a noninvasive, less costly, and efficient method that can be used for the diagnosis and follow up of patients with IBD (14).

Trans-abdominal US has been recommended as an efficient modality for IBD screening, specially for the as- sessment of anatomical extension of the CD when first diagnosed (15). A study by Hata et al. on patients with $\mathrm{CD}$, UC, and controls revealed a correlation between changes in the structure of different layers of the intestine and its pattern on abdominal ultrasound with different pathologies in patients with IBD using the total wall thickness and wall stratification. However, abdominal US findings were correlated with colonoscopic or other radiographic findings and disease activity. Besides, this study reported that CD and UC can be detected by US with a sensitivity and specificity of $86 \%$ and $89 \%$, respectively (16).

A meta-analysis of seven studies by Fraquelli et al., showed sensitivity and specificity of $75-94 \%$ and $67-$ $100 \%$ for US in the diagnosis of CD by assessing bowel wall thickness. However, they reported a sensitivity and specificity of $88.8 \%$ and $93 \%$ with a cut-off point of $3 \mathrm{~mm}$ for bowel wall thickness and sensitivity and specificity of $75 \%$ and $97 \%$ with a cut-off point of $4 \mathrm{~mm}$ for the diagnosis of CD (17).

A study by Pascu et al., on 61 patients with IBD comparing transabdominal US and MRI showed an accuracy of $89 \%$ for US and $73 \%$ for MRI in identifying active IBD. In this study patients underwent clinical and laboratory assessment, as well as ileocolonoscopy, transabdominal sonography, and MRI in five days. Positive result was defined as bowel wall thickness more than 3 $\mathrm{mm}$ and increased Doppler signal on US or contrast enhancement of the intestinal wall on MRI. However, both imaging methods showed significant correlation with the clinical disease activity(18) .

Moreover, in a systematic review by Panes et al., on 1029 patients from 5 studies reported overall sensitivity and specificity of $85 \%$ and $98 \%$ of US to assess CD (19).

There are debates on the correlation between indexes of clinical activity and biomarkers with US findings (19) . Studies have shown various results regarding the correlation between the clinical activity index and inflammatory markers (CDAI And Mayo) and bowel wall thickness (19). However, none of the studies were able to differentiate different intestinal wall layers and distinguish UC and $\mathrm{CD}$ especially in patients with indeterminate colitis (6). Besides, the role of US in UC patients has been less established in studies, though, there is less evidence in studies about the correlation between wall thickness and clinical disease activity (14).

EUS has been known as the best modality for assessing transmural changes in the bowel wall.

The type of EUS finding has been reported to be correlated with clinical severity and endoscopic grading and the extent of disease in patients with UC. Although preliminary studies using EUS to distinguish CD from UC were not a success, it was described as an efficient modality in the diagnosis of extra-intestinal findings (20).

Our study revealed a sensitivity of $100 \%$ and specificity of $90.9 \%$ for differentiating UC and $\mathrm{CD}$ compared to standard tests. We found significant correlation between TWT with Mayo score in patients with UC and between submucosal thickness with CDAI, ESR, CRP, and Calprotectin in patients with CD. Similarly, Shimizu et al. found 
a correlation between the increased thickness of intestine layers by EUS with active inflammation caused by IBD (21). While, Hurlstone et al. reported a strong correlation between inflammatory scores and wall thickness in rectal disease and described EUS as an adjunctive method for assessment of colonic wall in UC (20). A study by Dagli et al. showed a significant difference between TWT in patients with active or quiescent disease and controls. Transmural inflammation was reported in most CD patients while mucosal inflammation was seen in UC patients. Moreover, this study showed high accuracy of mucosal, submucosal and TWT thickness for differentiating active UC from remission and control group (11). Furthermore, Higaki et al. found a correlation between the mucosal and submucosal thickness of rectum in patients with relapsed UC compared to healthy controls using a mini-probe EUS (22).

Ellrichmann et al. in a study on 52 IBD patients and 61 controls reported $92.3 \%$ sensitivity for differentiating UC from CD by considering increased TWT, mucosal, submucosal thickness and presence of para-colonic lymph nodes. Besides, in this study UC patients had significant thickening of mucosa while CD patients showed significant thickening of Submucosa compared to healthy controls. Also, there was a significant correlation between TWT and inflammation scores; it was comparable to our study(6). These results were comparable to our results that revealed the sensitivity and specificity of $98.3 \%$ and $100 \%$ with a cut-off point of $2.75 \mathrm{~mm}$ for TWT in differentiating IBD from controls. Additionally, the sensitivity and specificity of TWT for differentiating CD from control was 97.1 and $100 \%$ with a cut-off point of $2.91 \mathrm{~mm}$ and for differentiating CD from UC and control was $70.6 \%$ and $58.3 \%$ with a cut-off point of $3.46 \mathrm{~mm}$. Correspondingly, the sensitivity and specificity of mean mucosal thickness for differentiating Ulcerative Colitis form Crohn's Disease and controls were $92.3 \%$ and $88.6 \%$ with a cut-off point of $1.1 \mathrm{~mm}$. While sensitivity and specificity of mean submucosal thickness for differentiating Crohn's Disease from Ulcerative Colitis and controls were 100\% and $86.1 \%$ with a cut-off point of $1.08 \mathrm{~mm}$.

\section{Limitation}

The main limitation of our study was our small sample size with an equal number of active and quiescent disease.

\section{Complications}

Our study showed no complication during the procedure due to the use of EUS in patients with UC, CD, and Controls.

\section{Conclusion}

According to our present results, Endoscopic Ultrasonography (EUS) can be used as an efficient modality with acceptable accuracy versus standard tests to differentiate Crohn's disease and Ulcerative colitis especially when a definite diagnosis cannot be reached by histopathology, imaging and colonoscopic results, clinical presentation, and laboratory findings. Additionally, it can be used for assessing patients' response to treatment during follow- ups with less cost and less invasiveness compared to regular colonoscopy or other radiographic devices. However, its accuracy is highly dependent on the experience level of the technician.

\section{Acknowledgments}

The research project number is 9311160038 approved by the Institutional Ethics Committee of Tehran University of Medical Sciences.

\section{Conflict of Interests}

The authors declare that they have no competing interests.

\section{References}

1. de Britto MAP, Soletti RC, Schanaider A, Madi K, de Souza HSP, Machado JC. Endoluminal ultrasound biomicroscopy as a reliable tool for in vivo assessment of colonic inflammation in rats. Int J Colorectal Dis. 2013;28(12):1613-20.

2. Baumgart DC, Sandborn WJ. Inflammatory bowel disease: clinical aspects and established and evolving therapies. The Lancet. 2007;369(9573):1641-57.

3. Guindi M, Riddell R. Indeterminate colitis. J Clin Pathol. 2004;57(12):1233-44.

4. Heresbach D, Alexandre J, Branger B, Bretagne J, Cruchant E, Dabadie A, et al. Frequency and significance of granulomas in a cohort of incident cases of Crohn's disease. Gut. 2005;54(2):215-22.

5. Van Assche G, Dignass A, Panes J, Beaugerie L, Karagiannis J, Allez $\mathrm{M}$, et al. The second European evidence-based consensus on the diagnosis and management of Crohn's disease: definitions and diagnosis. J Crohns Colitis. 2010;4(1):7-27.

6. Ellrichmann M, Wietzke-Braun P, Dhar S, Nikolaus S, Arlt A, Bethge $\mathrm{J}$, et al. Endoscopic ultrasound of the colon for the differentiation of Crohn's disease and ulcerative colitis in comparison with healthy controls. Aliment Pharmacol Ther. 2014;39(8):823-33.

7. Khaw K, Yeoman L, Saverymuttu S, Cook M, Joseph A. Ultrasonic patterns in inflammatory bowel disease. Clin Radiol. 1991;43(3):171 5.

8. Schwartz DA, Wiersema MJ, Dudiak KM, Fletcher JG, Clain JE, Tremaine WJ, et al. A comparison of endoscopic ultrasound, magnetic resonance imaging, and exam under anesthesia for evaluation of Crohn's perianal fistulas. Gastroenterology. 2001;121(5):1064-72.

9. Hildebrandt U, Kraus J, Ecker K, Schmid T, Schüder G, Feifel G. Endosonographic differentiation of mucosal and transmural nonspecific inflammatory bowel disease. Endoscopy. 1992;24(S 1):359-63.

10. Schwartz DA, Harewood GC, Wiersema MJ. EUS for rectal disease. Gastrointest Endoscopy. 2002;56(1):100-9.

11. Dağli U, Över H, Tezel A, Ülker A, Temuçin G. Transrectal ultrasound in the diagnosis and management of inflammatory bowel disease. Endoscopy. 1999;31(02):152-7.

12. Paine ER. Colonoscopic evaluation in ulcerative colitis. Gastroenterol Rep. 2014;2(3):161-8.

13. Sostegni R, Daperno M, Scaglione N, Lavagna A, Rocca R, Pera A. Crohn's disease: monitoring disease activity. Aliment Pharmacol Ther. 2003;17:11-7.

14. Strobel D, Goertz RS, Bernatik T. Diagnostics in inflammatory bowel disease: ultrasound. World J Gastroenterol. 2011;17(27):3192.

15. Parente F, Greco S, Molteni M, Anderloni A, Porro GB. Imaging inflammatory bowel disease using bowel ultrasound. Eur J Gastroenterol Hepatol. 2005;17(3):283-91.

16. Hata J, Haruma K, Suenaga K, Yoshihara M, Yamamoto G, Tanaka $\mathrm{S}$, et al. Ultrasonographic assessment of inflammatory bowel disease. Am J Gastroenterol. 1992;87(4).

17. Fraquelli M, Colli A, Casazza G, Paggi S, Colucci A, Massironi S, et al. Role of US in detection of Crohn disease: meta-analysis. Radiology. 2005;236(1):95-101.

18. Pascu M, Roznowski A, Müller HP, Adler A, Wiedenmann B, Dignass A. Clinical relevance of transabdominal ultrasonography and magnetic resonance imaging in patients with inflammatory bowel disease of the terminal ileum and large bowel. Inflammat Bowel Dis. 
2004;10(4):373-82.

19. Panes J, Bouzas R, Chaparro M, García-Sánchez V, Gisbert J, Martínez de Guereñu B, et al. Systematic review: the use of ultrasonography, computed tomography and magnetic resonance imaging for the diagnosis, assessment of activity and abdominal complications of Crohn's disease. Aliment Pharmacol Ther. 2011;34(2):125-45.

20. Lew RJ, Ginsberg GG. The role of endoscopic ultrasound in inflammatory bowel disease. Gastrointest Endosc Clin N Am. 2002;12(3):561-71.

21. Shimizu S, Tada M, Kawai K. Endoscopic ultrasonography in inflammatory bowel diseases. Gastrointest endosc clin N Am. 1995;5(4):851-9.

22. Higaki S, Nohara H, Saitoh Y, Akazawa A, Yanai H, Yoshida T, et al. Increased rectal wall thickness may predict relapse in ulcerative colitis: a pilot follow-up study by ultrasonographic colonoscopy. Endoscopy. 2002;34(03):212-9. 